\title{
New Generating Technique for Image Steganography
}

\author{
Seifedine Kadry and Sara Nasr
}

\begin{abstract}
Steganography is used to send secure data hidden in image or another type of media in order to prevent intruders from detecting it. Image steganography depends on existing images so that data bits may be added to the image bits or replace some bits of it, the goal is to hide the bits of a secret text behind image bits so that no one other the sender and the receiver can discover what this file include beneath the cover image.

Our developed 'New Generating Technique' (NGT) is based on generating the image from the text that we want to send securely. This means no additional data is added to the initial text it is just a change in its format so that we can send it safely. This may be done using different image formats starting with grayscale images to 24 bits. The most important advantage in our proposed steganographic technique is that it does not require an additional image to hide the text beneath it, besides it has a small size and very fast in comparison with other techniques.
\end{abstract}

Index Terms-Steganography, image, security, cryptography.

\section{INTRODUCTION}

Nowadays and because of unsecure networks and internet that can be accessed by anyone it became very risky to send important messages and files without any security measures. Before sending an important message we have to make sure that it will arrive to the destination in a secure way without being seen or modified by an intruder. Because of the threats mentioned before, steganography was discovered. Steganography in brief is the art of hiding information by other information. Usually we need to hide a text beneath any type of media. The most type of media used in hiding text is image. Steganography can be considered a new technology that still needs work and improvements. We are interesting in image steganography base type because it is s very good idea according to a security method to send an image that doesn't draw any attention or suspicions about containing an important message. Another important reason for our choice was the use of this type of security by terrorists to hide their plans so everyone should read and be aware of such subjects. Steganography is one of the newest technologies in sending secure data between sender and receiver. After the large spread of cryptography a new concept was added in order to add a new security level that prevents the detection of hidden information under a cover of any digital media. Nowadays, the internet became a robust and enormous world that offers a

Manuscript received January 15, 2013; revised March 25, 2013.

S. Kadry is with the American University of the Middle East, Kuwait (e-mail: skadry@gmail.com).

S. Nasr is with the Arts Sciences and Technologies University, Lebanon (e-mail: saranasr85@gmail.com). diversity of facilities and needs, because of these different functionalities it became easier to find diversity of ways to send messages to people without anyone noticing or even knowing that the message exist. Steganography is the procedure in which one type of communication (text, sound or image beneath another type. Steganography is described by Neil F. Johnson and Sushil Jajodia in their paper 'Steganalysis: The Investigation of Hidden Information' [1] as 'The goal of Steganography is to avoid drawing suspicion to the transmission of a hidden message. If suspicion is raised, then this goal is defeated.' This technology is used widely nowadays, and a lot of people started hiding data, images video and audio by using it. For this reason even if a message seems normal and innocent it might be holding within it a secret message with high important data.

The source of the word Steganography comes from the Greek word steganos which means covered or secret and the word graphy which means writing or drawing. So the whole meaning is secret writing [2].

To clarify the idea of steganography, the three famous characters named Alice, Bob and Ward are used. Alice (A) wants to send a secret message (M) to Bob (B). Bob must receive it safely without raising suspicion. To do that, Alice changes the message (M) into a steganography object (stego-object, i.e. new file carrying the embedded-object) (S). Stego-object is created by covering the message (M) with another random harmless message to produce a cover $(C$, i.e. data file that will hold the secret message). Covering the massage (M) with message (C) happens by using a secret key (stego-key) (K). Now Alice should be able to send the stego-object (S) to Bob without being detected by Ward. When Bob receives $(\mathrm{S})$ he will use the stego-key $(\mathrm{K})$ which he already knows to reproduce secret message (M) from the cover message $(\mathrm{C})$ and be able to read it. Steganography have to guarantee these requirements [3]:

- Robustness: information is robust when it is embedded inside an image and although it disappeared behind it but it is not destroyed, it is present, but is only detected with reliability after modifying the image.

- Undetectability: the data hidden under an image cannot be detected as long as the cover image is not doubtable or suspicious and looks unchanged.

- Perceptual transparency: this requirement depends on human visual and audio system. If the hidden data didn't raise the attention of human systems and no one could distinguish whether the cover contains secret data then this requirement is guaranteed.

- Security: as long as no one other than the legal receiver can remove the embedded data from behind cover, the embedding algorithm is said to be secure. This requirement assures that no targeted attacks can detect or view the hidden message unless they have a full knowledge of the 
embedding algorithm.

There may be different approaches for steganography methods classification. They may be grouped according to the type of cover file or according to the different modifications each method may apply during embedding process. Our proposed technique belongs to the second classification type. We can find more than six different methods concerning the second classification, noting that in some cases it is very difficult to classify a specific method one clear category. Here are the six most used methods: Substitution, Injection (or insertion), Distortion, Generation, Transform domain techniques, Statistical steganography [4].

\section{Proposed TeChNiQue}

Our Generation method differs from other steganography methods in concept because it doesn't require an existing cover file. It is a technique that creates a cover file for the only one reason of hiding the sent message. The biggest advantage of this method is that no one can compare the sent image with another already existing image, using a generation approach the result is always an original file. This file of course has a huge strength against comparison tests.

As we said generating technique doesn't depend on an already existing image file for this reason we will use the data file itself in order to create or generate a cover file. At the same time, the data that we'll send will be hidden in the cover. We have used the text bits in order to create a new sequence of bits that represents different colors in addition to a common key that the sender and receiver agree on. This main concept of steganography can produce different types of images depending on the way we deal with bits. As a result of our research we will have two main new techniques of steganography. The first technique that will be explained is generating an image of 8 bit grayscale colors from the original text, while the second technique is generating an RGB image from the original text. This RGB image is a 24 bit image that is generated from the original text.

\section{A. Gray Scale Generating Image Technique}

We will study the gray scale case only. Gary scale colors are degradation of 256 colors varying between white and black. Each 8 bits combined together represent a different color. After taking the original text that the sender needs to send privately to a specific destination, the sequence of text bits can be XORed with a secret key used for steganography goals. Of course the key size is most of the times different from the size of the text in this case the key will be repeated till its size reaches the size of the text. I this way we obtain a polyalphabetic cipher text. After XORing the bits of text with the resized key bits, a new sequence of bits will be generated. So the text bits themselves are now changed in to a new sequence of bits. After that the new bits will be divided into groups of 8 bits (one byte). The reason for this grouping is to have a set of bytes each byte representing one color out of the 256 grayscale colors (numbered 0 up to 255 or 00 till $\mathrm{FF}$ in hexadecimal). Now instead of having a sequence of bits representing letters of a text these bits after being xored with a repeated or resized secret key, they represent now different colors of grayscale (Fig. 1).

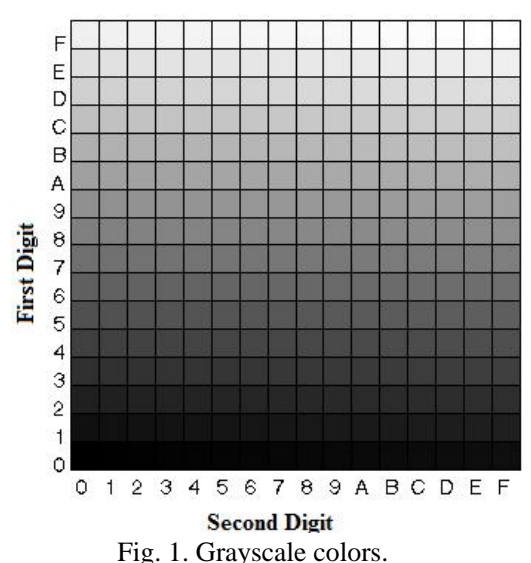

Example: Giving a brief example will help us explain our idea and make it clearer: We will take a part of the bit sequence corresponding to the original text, knowing that every 8 bits are in ASCII code are the representation of a letter from the text. Below is a table (Table I) of the ASCII code representations of the characters that we will use in the example:

TABLE I: ASCII CODE OF "THESIS"
\begin{tabular}{|c|c|c|}
\hline Binary & Dec & Glyph \\
\hline 01010100 & $\mathbf{8 4}$ & T \\
\hline 01101000 & 104 & H \\
\hline 01100101 & 101 & E \\
\hline 01110011 & 115 & S \\
\hline 01101001 & 105 & I \\
\hline 01010011 & 83 & S \\
\hline 01110010 & 114 & R \\
\hline 0100000 & 32 & space \\
\hline
\end{tabular}

Original text:

Thesis

010101000110100001100101011100110110100101110011

Key of four letters only:

Sara:

01010011011000010111001111100001

\section{B. Encode}

We will XOR the original bits with the key. As we said the key must be resized, so we must repeat the key till the bits of the text are all xored. In our example the key sequence will be repeated 3 times:

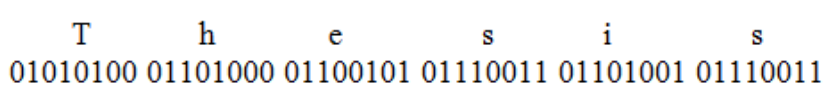

XOR

$\begin{array}{llllll}\text { S } & \text { a } & \text { r } & \text { a } & \text { S } & \text { a }\end{array}$ 010100110110000101110011111000010101001101100001

000001110000010000010111000100100011101000010010

In hexadecimal the bits are:

$\begin{array}{llllll}7 & 4 & 17 & 12 & 3 \mathrm{~A} & 12\end{array}$


This result will be presented in a sequence of 6 different colors of the gray scale table colors (Fig. 2):
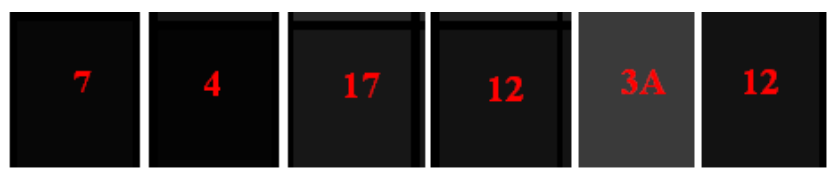

Fig. 2. 6 different colors.

Output display: suppose the output is a large collection of colors, of course we cannot display them on the same line all. We must find a method in order to show the output as an image of rectangular or square shape. In order to show the sequence of colors displayed on the screen in a correct way we have used a function that finds the nearest square root and shows the output as a square image. Let us take our example above we have 6 different colors, the nearest larger number that returns an integer square root is 9 . The square root of nine is 3 . So if we had 9 colors it may have been easier to show every 3 colors on a separate line. In our source code we have appended 3 additional bytes to the original text that represents the space of value 32 which represents 00100000 byte value.

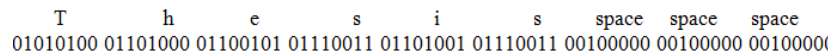

XOR

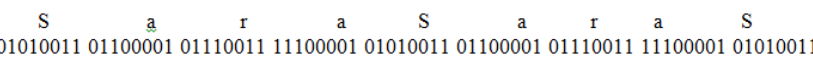
000001110000010000010111000100100011101000010010010100111100000101110011

In hexadecimal the bits are:
7
$4 \quad 17$
$12 \quad 3 \mathrm{~A}$
1253
C1 73

The displayed image (Fig. 3) will be as follows:

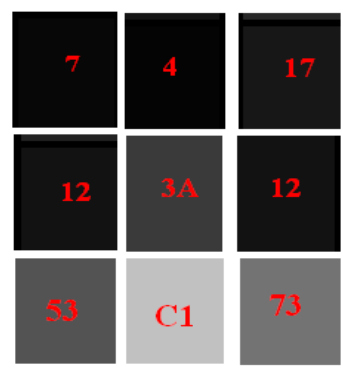

Fig. 3. Square of 9 colors.

The decoding process is the opposite of the coding process. Following the flowcharts of encoding and decoding process: Encode (Fig. 4):

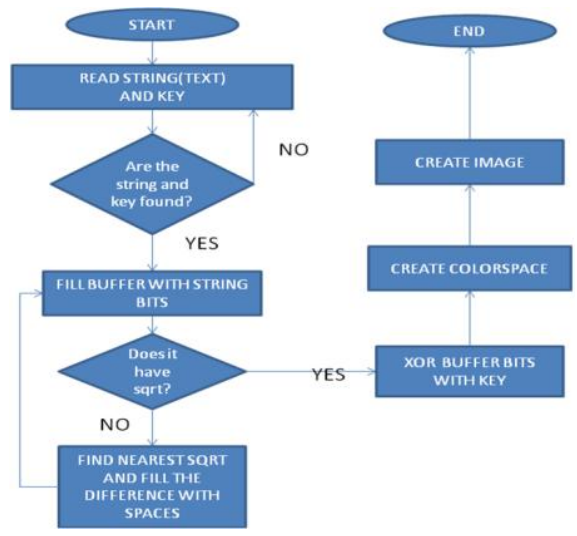

Fig. 4. Encoding flowchart.
Decode (Fig. 5):

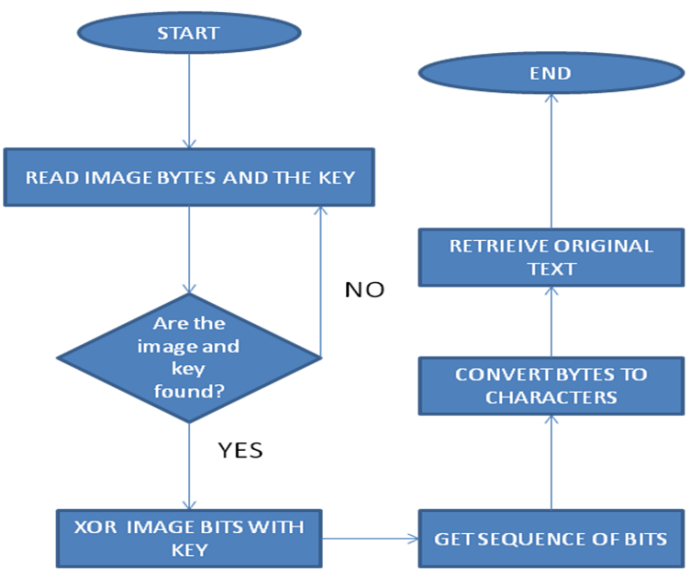

Fig. 5. Decoding flowchart.

\section{Advantages of the Proposed Technique}

The most important advantage in this steganographic technique is that it does not require an additional image to hide the text beneath it, besides it has a small size in comparison with all other techniques because no additional bits or data is added to the encrypted file so the receiver receives the file with same size as the original text (Table II). Additionally, our technique is polyalphabetic because when a letter of the original text is changed in to a color it is not necessary to have the same color in the entire image, on the contrary the diversity of colors that will appear encoding the same letter gives our method more strength against steganalysis.

\section{Disadvantages of the Proposed Technique}

In this stage of implementation it is still weak against attacks and still raises the attention of intruders but the suggestion that will be mentioned in the future work section, to improve this idea will help in maintaining a better steganographic technique that is more secure and not suspicious.

\begin{tabular}{|l|l|l|}
\cline { 2 - 3 } \multicolumn{1}{c|}{} & \multicolumn{1}{c|}{ TABLE II: INVISIBLE SECRET 4 V/S NGT } \\
\hline Original text secrets 4 & \multicolumn{1}{c|}{ NGT software } \\
\hline $\begin{array}{l}\text { Image } \\
\text { carrier }\end{array}$ & $\begin{array}{l}900 \mathrm{~KB} \text { (to use carrier of } \\
26 \mathrm{~KB} \text { we need text of size } \\
9720 \text { Bytes) }\end{array}$ & N/A \\
\hline Received file & $925 \mathrm{~KB}$ & $25 \mathrm{~KB}$ \\
\hline $\begin{array}{l}\text { Encoding } \\
\text { Time }\end{array}$ & 2.06 seconds & 0.49 second \\
\hline
\end{tabular}

\section{ANALYSIS OF THE PROPOSED TECHNIQUE}

The first and most important advantage of NGT in comparison with old methods is its small size, because there's no additional bits added to the original file. Another advantage is the simplicity in creating the cover file because this technique doesn't require two different file for data and image.

LSB (Least Significant Bit) [5], [6] is considered the fastest technique between old methods and has a lot of 
advantages with respects to its small size and its simplicity and fastness.

TABLE III: LSB V/S NGT

\begin{tabular}{|l|l|}
\hline \multicolumn{1}{|c|}{ LSB } & \multicolumn{1}{|c|}{ NGT } \\
\hline $\begin{array}{l}\text { cannot be noticed easily by } \\
\text { the human being naked eye }\end{array}$ & $\begin{array}{l}\text { Collection of colors can } \\
\text { arise doubts }\end{array}$ \\
\hline $\begin{array}{l}\text { Larger size of image for same } \\
\text { original text file }\end{array}$ & $\begin{array}{l}\text { Smaller size of image for } \\
\text { same original text file }\end{array}$ \\
\hline $\begin{array}{l}\text { Takes time to finish steps of } \\
\text { encoding because a cover } \\
\text { image is used }\end{array}$ & $\begin{array}{l}\text { Faster because only text file } \\
\text { is required }\end{array}$ \\
\hline $\begin{array}{l}\text { 1 bit of the original text is } \\
\text { encoded within each byte and } \\
\text { maximum of 3 bits per byte }\end{array}$ & $\begin{array}{l}8 \text { bits encoded within each } \\
\text { byte. }\end{array}$ \\
\hline
\end{tabular}

There's a big variety of software that use LSB method, one of these famous software is the Invisible secret. 'Invisible secrets' has been always updated with new versions. We have taken Invisible secret 4 (http://www.invisiblesecrets.com) in order to make a comparison between it and between our NGT software (Table 3). Invisible Secrets 4 is a powerful security suite that can hide and encrypt files, also can destroy internet traces, shred files, make secure IP-to-IP password transfer and even lock any application on the computer. It is an easy to use with a powerful wizard interface. It was first released in 1999; the latest version is -Invisible Secrets 4 that was released in 2009 by NeoByte Solutions. Invisible secret 4 uses the three lest significant bits to hide text. This software also includes cryptographic methods that can be added in order to give more security to the sent message. But we didn't use these cryptographic methods to be fair and compare pure steganographic methods with each other. We even used same type of images (BMP). We calculated the time taken to encode and the size of text files and the size of carriers.

\section{CONCLUSION ANd PERSPECTIVE}

In this paper, we developed a new generating technique for image steganography, which is works on the bits of the original text to create and image, without using a separate cover image, of same size and by the help of a secret key. The advantages of this technique are the small size of image it produces and the fast way it works in.

Although this technique has advantages that we already stated including small size and fast execution, but it has also some disadvantages and weakness that can be avoided in future improvements. Some of these improvements:

- Replacement of 8 bit gray scale color: since this technique will produce 8 bit gray scale color, in the future improvements it can produce instead of 8 bit gray scale colors another colors such as: 8-Bit Palette colors and 16-bit grayscale color.

- Drawing different shapes: the output of this generating technique is an image of square shape. Some suggestions that can make improvements to this steganography methods is to write name or design images (hand drawn shapes, names, images) instead of having all images of same square shape. The sender can draw any shape he wants the shape will be filled with colored pixels that the text will use while the exterior of the shape will be ignored.

- Random pixels distribution: To make our steganographic file harder to break we can change the distribution of the pixels. Each 8 bits or byte can be given a unique number this number sets the original position of each byte. After having all bytes given unique positions, the place of the bytes may be changed randomly.

- Create a meaningful image of the resultant squares

\section{REFERENCES}

[1] N. F. Johnson and S. J. Steganalysis, "The Investigation of Hidden Information," IEEE Information Technology Conference, Syracuse, New York, USA, September 1-3, 1998, pp. 113-116.

[2] Information Hiding Using Steganography. (2003). Lecture notes, Department of Computer System \& Communication Faculty of Computer Science and Information system University Technology Malaysia. [Online]. Available: http://www.lia.deis.unibo.it/Courses/RetiDiCalcolatori/Progetti98/For tini/cover.html

[3] A. A. Abdelwahab and L. A. Hassaan, "A discrete wavelet transform based technique for image data hiding," Radio Science Conference, 2008, Egypt, pp. 1-9.

[4] S. Katzenbeisser, in Information Hiding Techniques for Steganography and Digital Watermarking, Fabien and A. P. Petitcolas ed., Artech House, December 31, 1999.

[5] J. J. Roque and J. M. Minguet. "SLSB: improving the steganographic algorithm LSB," Ibero-American Congress on Information Security (CIBSI), 2009, Uruguay.

[6] R. Chandramouli and N. D. Memon. "Analysis of LSB based image steganography techniques," in Proc. IEEE ICIP, vol. 3, 2001, pp 1019-1022.

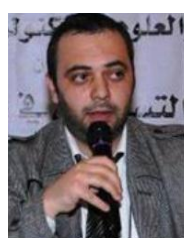

Seifedine Kadry is an associate professor at the American university of the Middle East, Faculty of Engineering, KUWAIT. He got his Master Degree in Computer Science and Applied Math from AUF-EPFL-Inria, Lebanon in 2002. He received the Doctor degree from the Clermont Ferrand II University, France in 2007. His Research interests include software testing and security.

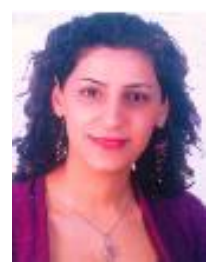

Sara Nasr is a graduate student in the computer science and communication department at the Arts, Sciences and Technologies University in Lebanon. Her research interests include security, cryptography and steganography. 